\title{
Opportunities for the Use of Business Data Analysis Technologies
}

\author{
Signe Bālina ${ }^{1}$, Rita Žuka ${ }^{2}$, Juris Krasts ${ }^{3}$ \\ ${ }^{1-3^{3}}$ University of Latvia
}

\begin{abstract}
The paper analyses the business data analysis technologies, provides their classification and considers relevant terminology. The feasibility of business data analysis technologies handling big data sources is overviewed. The paper shows the results of examination of the online big data source analytics technologies, data mining and predictive modelling technologies and their trends.
\end{abstract}

Keywords - Analytics platforms, business analytics, business intelligence, data mining, online analytical processing, predictive modelling.

\section{I.INTRODUCTION}

The accumulated data volume is steadily growing in enterprises and organisations, and the amount of data increases exponentially. In 2015, the researchers of Unisphere Research emphasised that $90 \%$ of all data in the world was created in the last two years and this process was strongly influenced by the Internet. Enormous amounts of different data have been created. The data is stored in different data sources (relational databases, non-relational databases - NoSQL databases, spreadsheets, text documents etc.). Some data, such as journals, history lists, videos, text documents, is stored in a poorly structured format that does not meet traditional database relational models. IBM emphasises that $80 \%$ of information created and used by the enterprise is the unstructured data (IBM, 2015). The goal of this paper is to consider the usage and tendencies of the business data analytics technology, terminology and the proposed business data analysis tools.

The business data analysis technologies have been developed for the accumulated data analysis, which is rapidly developing. Only a short time ago, in the business analysis the structured data was used, and the task of an analyst (or a business user) was to get the answers to pre-formulated questions and later make the corresponding report. Nowadays, the job of an analyst or a business user is an interactive process, involving the search for hidden relationships and patterns in the data. It is a process where the raw data are transformed into useful information. To enable business data users to access their large volumes of data, there is a need for special software packages or applications, which search for the relationships between the data, and this ensures more well-grounded decision-making.

The range of business data analysis tools is very wide, and recently the Business Intelligence (BI) and Analytics Platforms (AP) market is experiencing significant changes. The requirements for the BI tools are being changed. Business users are increasingly demanding tools with an interactive user interface, which provides data analysis without any special knowledge of information technology requirements (Sallam et al., 2015).
In both the recent scientific literature and web publications related to business data analysis technology, different terms are used: Business Intelligence, Business Analytics (BA), Analytics Platforms, Online Analytical Processing (OLAP), Data Mining (DM), Advanced Analytics, Visual Data Discovery and Big Data (BD).

\section{Business Intelligence}

Business intelligence is a very broad term. Gartner provides the following definition: "Business intelligence (BI) is an umbrella term that includes the applications, infrastructure and tools, and best practices that enable access to and analysis of information to improve and optimize decisions and performance" (Gartner Group, 2015).

The first generation of BI applications ensured the selection of data from relational databases to online transaction processing systems (OLTP) as well as the data transformation, thus creating the multidimensional databases - the data warehouses. Data warehouses store aggregated data from transactional databases. Gartner defines a data warehouse as "a storage architecture designed to hold data extracted from transaction systems, operational data stores and external sources" (Gartner Group, 2015).

Data for analysis is often stored in different data sources: in different transaction systems. The OLTP systems provide simultaneous execution of a large number of short transactions by multiple users, data collections and editing. The speed and reliability of performance for the transaction update is important for the OLTP systems. But the OLTP systems are not focused on analytical data processing. Data is distributed across multiple tables and their aggregation is the performance of complex operations.

Storage and processing of the data are only some of the tasks as the data must also be converted into the structure suitable for analysis and giving useful information. The founder of the relational model, Codd pointed out the shortcomings of the relational model. Codd (1979) proposed to transform the data from the relational model to the multidimensional structure in order to facilitate data analysis.

There are different multidimensional database structures, such as OLAP cubes and databases with associative architecture.

\section{OLAP and Associative Architecture}

Online analytical processing systems have been developed specifically for the data analysis. By Microsoft experts, the term "OLAP" is associated with the specialised tools and the technology providing a multidimensional model of data storage in the form of multidimensional cubes. OLAP cubes use a special multidimensional data model, which allows more efficient use 
of the data collected from the operational systems and the data warehouses (Microsoft, 2011).

IBM experts define OLAP cube as "the multidimensional database that is optimized for OLAP applications" (IBM Corporation, 2013). There are three main components of OLAP cube: dimension (the attributes of the object which being analysed), member (a specific value of an attribute) and measure - fact (aggregated data, parameter to analyse) (IBM Corporation, 2013).

The multidimensional structure of the data with the possibility of applying different levels to the data detail is a natural way of the data analysis for companies and organisations (Oracle, 2014).

OLAP functionality has been implemented in various ways, starting with the simplest office applications and ending with the distributed analytical systems based on server products.

The data source for the OLAP cubes is usually a relational database. Analytical services do not include tools to replenish relational data warehouse from the operational database. Such tools contain many modern server databases. Microsoft offers Microsoft SQL Server Integration Services (SSIS). This is a server tool to ensure migration of the data from one relational database management system (RDBMS) to another with the possibility of transformation, which can also be used to replenish data warehouse. Integration services can be used not only with Microsoft SQL Server, but also with any other data sources available through a universal mechanism to access the OLE database data (Microsoft, 2015).

Thus, OLAP supports a flexible view of the information, arbitrary slices of the data, different levels of detail, data consolidation, rotation, comparison according to the dates, time series analysis.

OLAP systems are not equipped with the supported opportunity of forecasting the data. Inductive inference is a characteristic of data mining (Oracle, 2014). OLAP is more suitable for understanding the historical data, while the data mining uses historical data to answer questions about the future.

An alternative approach to the storage and processing of information is implemented in systems with associative architecture. In such systems, handling and storage of all the information are implemented in the memory (in-memory). The memory stores the individual data tables and associative links between them. Each value of each field is associated with all the other values in the entire database. Such systems use an associative data model. The associative model is automatically generated during the process of loading data by linking the key fields of tables and simultaneously creating relations between the table fields with the same names. For the first time, this architecture has been implemented in QlikView (Qlik, 2014).

\section{Data Mining}

Oracle defines data mining as "the practice of automatically searching large stores of data to discover patterns and trends that go beyond simple analysis" (Taylor, 2014). Data mining uses a model for data analyses. The model is based on the data stored in relational databases and data warehouses, and it can be applied both to new data and the data where it is built. Traditional statistical methods of data analysis or the usage of OLAP technology are mainly focused on pre-test formulated hypotheses. One of the main tasks of data mining is the search for non-obvious patterns. Data mining tools can find these patterns and their own hypotheses regarding the relationships.

The process of building the data mining model includes several stages. Oracle experts have defined seven stages of business data analysis process:

1. definition of requirements: the understanding of business process objectives and requirements, the definition of analysis target;

2. data acquirement: data collection and preparation for further analysis;

3. data understanding;

4. manipulation of data: the data filtering, elimination, as well as removing of incorrect data;

5. data publishing;

6. data analyses: model corresponding to business process and given data, model estimation and learning;

7. decision making and taking action: model evaluation, testing, comparison, and application of the model (Oracle, 2014).

The stages of model building and evaluation process are shown in Fig. 1.

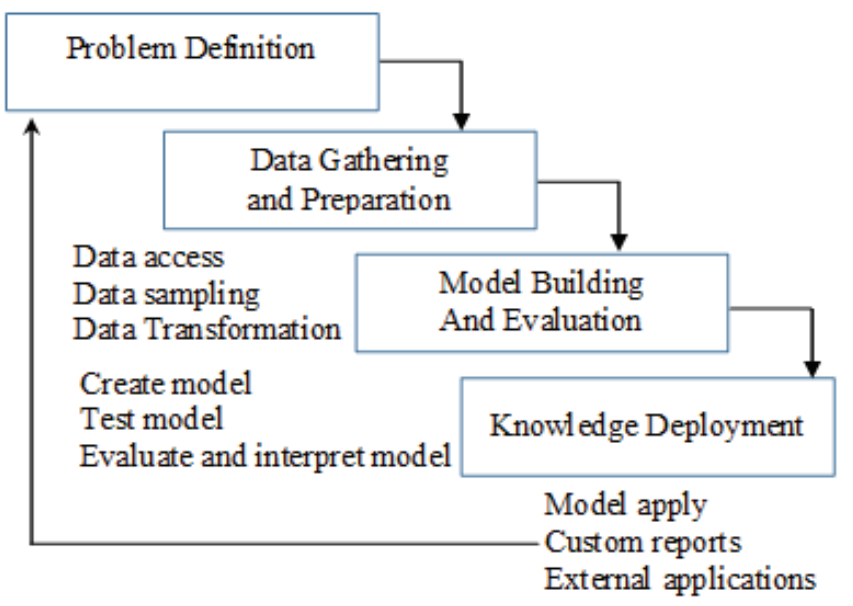

Fig. 1. Model building and evaluation (Kathy L. Taylor, 2014).

Oracle experts (2014) have stressed that for a large volume of data, the data preparation, cleaning and creating multidimensional database structures can take $80 \%$ to $90 \%$ from the analysis itself. The data preparation is very important for the data analysis, because multidimensional database can be deemed useful if it contains correct and sufficient data for solving specific tasks. The data analysis results depend on the quality of the data.

The data mining algorithms are sensitive to outliers. The understanding of the analysed data is crucial for the usage of data mining algorithms. At first, it is necessary to select factors (or predictors), after that the model has to be trained based on their usefulness for analysis. Therefore, the understanding of the data is a necessary condition for the correct interpretation of the analysis results. Overloading the model with predictors not related to the task can lead to deterioration of the detection of ability model. 
At the stage of model building, BI software products support methods how to evaluate each factor's influence on the dependent variable. For example, predictor evaluation can be performed automatically by using Microsoft Analysis Services, where the importance scores of each predictor are calculated (see Fig. 2).

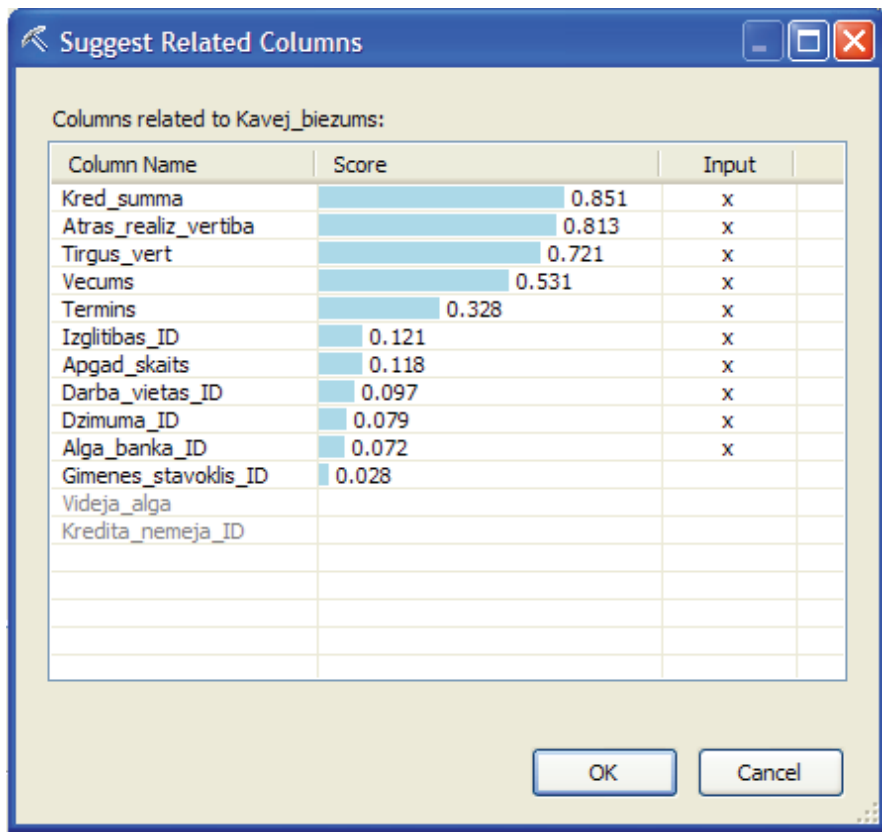

Fig. 2. Score calculation results of influence of different factors using Microsoft Analysis Services.

The model evaluation process involves the checking of model accuracy. The classification matrix is one of the tools for evaluation of model accuracy. Data are separated into training and testing sets. Samples of various sizes can be used for testing, and the accuracy of the model increases along with an increase in the size of the data set. The model adequacy is checked by the analyses of specific properties of the model that are significant in terms of the ongoing investigation. Assessment of the results obtained from the models requires an analyst's knowledge in the subject area in order to correctly analyse and interpret the identified influence and patterns.

Big data stimulates the usage of more extensive data mining techniques. This is due to the fact that an enormous volume of the information varies in nature and content (Brown, 2012).

Data mining allows for two types of business process modelling: analysis and forecasting. Descriptive modelling makes it possible to analyse the data sets that will not be complemented, and offers the opportunity to create the process model that is based on the given set of data. Predictive modelling makes it possible to estimate a model that works with the new data. Predictive modelling is based on the usage of the estimated descriptive model.

The data mining technology uses statistical and cybernetic methods of the data analysis, such as classification, modelling and forecasting, as well as statistical methods such as descriptive analysis, correlation and regression analysis, factor analysis, variance analysis, component analysis, discriminant analysis, time series analysis etc. Data mining technology combines formal methods that are based on quantitative data analysis with informal methods based on qualitative analysis of data. Although the data mining uses statistical methods, there are differences between the data analysis methods in data mining and traditional statistical methods. The usage of statistical methods and the building of statistical models are generally based on the assumption concerning the data distribution. If these assumptions do not perform, the analysis cannot be not used. The data mining technology uses statistical methods, but does not require assumptions on the nature of the data (data distribution), and, therefore, the model is not built on such assumptions. For example, machine learning methods make weak assumptions about the nature of the original data. The knowledge gained through the data mining methods is usually presented in the form of models; in addition, models provide visual representation of the results, which makes them accessible for use even without having the special mathematical background (Taylor, 2014).

The data mining technology supports a wide range of methods and algorithms. In general, modelling methods are divided into the following categories:

The association algorithm is applied to identify the patterns between the related events, objects and their attributes. The method is based on finding the correlation between the different attributes of the object.

The classification algorithm is a method of the data mining, which defines the category or class for a specific object instance. The classification problem is a discrete task.

The anomaly detection algorithm is one of the segmentation methods. The method allows for detection of abnormal data in homogeneous data. The method is used for detecting the fraud (Taylor, 2014).

The clustering (segmentation) algorithm provides the detection of independent object segments (clusters) and object pooling into clusters based on object-specific attributes called factors. The clustering algorithm groups together the objects having many similar attributes. The objects attached to a specific segment are similar to attribute values. In the model learning process, there is not some particular single attribute used, but all presented object attributes are used to an equal extent. The clustering algorithm calculates the model through successive iterations, and the gradual approximation ended when the segment (cluster) border came closer to the final result.

The regression algorithm performs variable value estimation using distinctive attributes (dependent factors). Regression models can be estimated using not only quantitative attributes, but also qualitative ones. The most popular ones are linear regression and logistic regression models.

The sequential pattern method is used to calculate a model that helps analyse and predict sequence of events in time.

The choice of algorithm depends on the aim of a business task.

Different software providers offer different data mining algorithms. For example, algorithms provided by Microsoft Analysis Server and IBM SPSS Modeler are shown in Fig. 3 and Fig. 4, respectively. 


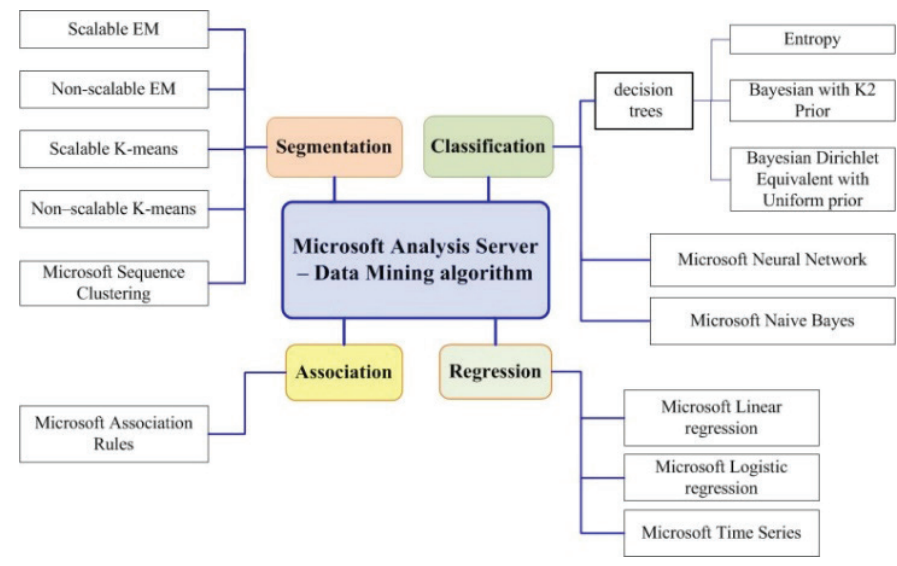

Fig. 3. Analysis Server data mining algorithms (Microsoft, 2015b).

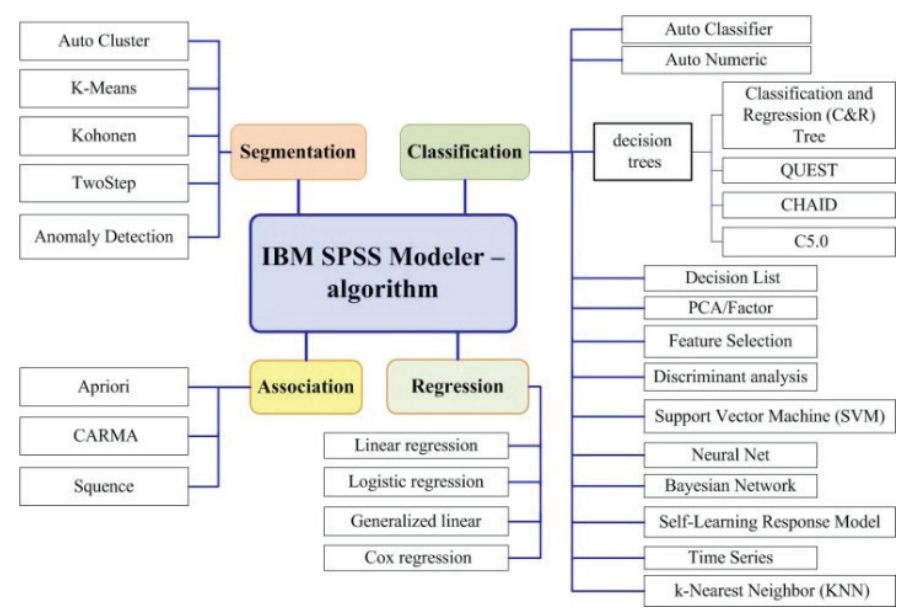

Fig. 4. IBM SPSS Modeler data mining algorithms (IBM Corporation, 2011).

Data mining technology involves the comparison of different models. BI software supports several methods for the selection of the best model. Many of them are based on a comparative evaluation of the models. The process consists in applying different models to the same sample of data and then comparing characteristics of models. The comparative evaluation of data mining models can be performed by means of evaluation charts, such as Profit Chart, Gains Chart and Lift Charts (see Fig. 5).

The process of the development of model is not fully completed after the finding of the best model. Periodically, it is necessary to evaluate the adequacy of the model to a new data set. New data and new models can cause additional questions and the need of improvement.

\section{BI and Advanced Analytics Platforms}

Development of an analytical platform was due to the need to facilitate the process of data analysis. The analytical platform includes all the tools for each phase of the process of search and retrieval of patterns of "raw" data. Data analysis platform comprises tools for data extraction and transformation, tools for data consolidation to the data warehouse. It contains tools to support and use data mining algorithms, as well as the visualisation and dissemination of results.

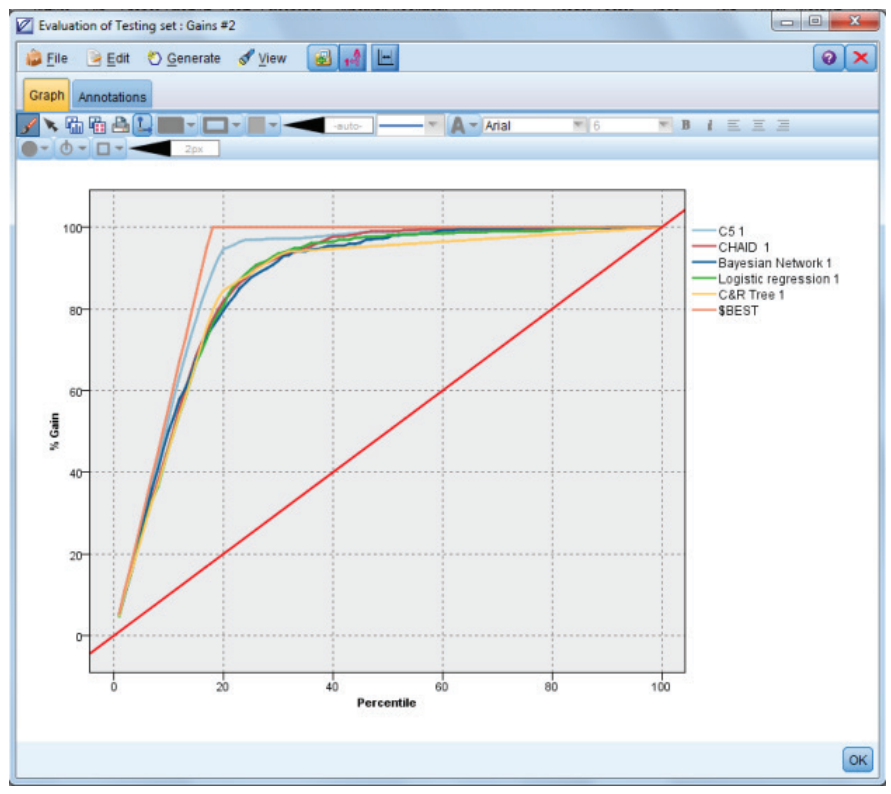

Fig. 5. Evaluation of model characteristics by Gains Chart using IBM SPSS Modeler.

Further spread of BI technologies and increased demand for business data analysis led to the creation and development of an advanced analytics platform.

An advanced analytics platform is software that provides data users with "full-spectrum analytics from data exploration and discovery through advanced and predictive analytics" (Oracle, 2014).

According to Gartner, an advanced analytics platform must use special quantitative methods of data analysis, such as statistics, descriptive and predictive data mining, simulation and optimisation that achieves results inaccessible by a traditional BI technology (Herschel, Linden, Kart, 2015).

Based on the research by Gartner, leaders for advanced analytics platforms were SAS, IBM, KNIME and RapidMiner (Herschel, Linden, Kart, 2015).

SAS is a leader in the development of the BI software over the years with $35.4 \%$ market share in advanced analytics (SAS, 2015). SAS provides a wide range of products and analytical techniques available through programming by the open-source environment R. One of the advantages of SAS platform is availability of analytic applications focused on solving specific business problems.

The high position of IBM was achieved through the acquisition of the SPSS Statistics and SPSS Modeler that can be integrated with the platform Watson. Gartner notes that IBM success is associated with a deep understanding of the needs of its customers, quick response to changing market trends and the provision of BI tools aimed at different levels of readiness of customers, provision of business-friendly tools. (Herschel, Linden, Kart, 2015).

RapidMiner is a complex system for data mining that also provides processing of big data statistical analysis. The success of KNIME and RapidMiner is explained by the fact that along with the licenced versions of their products, they provide also free versions. Products are distributed as an open source; they provide a desktop-based advanced analytics platform, server-based on-site or customer cloud solution. 
By Gartner, Microsoft is included in the "visionaries" group. Predictive analytics functionality is implemented by using Microsoft SQL Server Analysis Services (SSAS) that are embedded within Microsoft SQL Server. Customers gave a low assessment of SSAS, but Microsoft's new Azure Machine Learning (AML) cloud was still on beta stage at evaluation time (Herschel, Linden, Kart, 2015).

Oracle has been excluded from the Gartner Magic Quadrant. Gartner explains the exclusion of companies with the fact that Oracle advanced analytics tools cannot be considered standalone products: they are available only in the form of tightly integrated modules, Oracle database, BI and big data (Herschel, Linden, Kart, 2015).

By Gartner estimation, the amount of advanced analytics segment is over \$ 1 billion. The further growth is associated with the growing need for more data and the flow of information from different data sources. The market of business analysis is changed in accordance with the needs of business users (Herschel, Linden, Kart, 2015).

Gartner notes that the growth of data, new varieties of data type, growth in the number of users make new demand to the level of complexity of BI systems. The number of users for which the system must be specifically adopted is also increasing - they are expecting intuitive analysis tools for getting quick results. Thus, Gartner predicts growth of demand for self-service tools (Herschel, Linden, Kart, 2015).

Gartner research shows that the largest part in the demand of new $\mathrm{BI}$ products is due to the data discovery, decentralisation, and empowerment of business users. Given the current market trends, new BI vendors have appeared who specialise in the creation of BI tools with a visual and highly interactive user interface. At the same time, traditional BI platform vendors, such as Microsoft, IBM, SAP, complement their platforms by the tools aimed at a simplified and interactive visual data analysis (Sallam et al., 2015).

There is a move to a platform that can be used by analysts and ordinary business users to accept and justify business decisions and which differs in rapid implementation. This shift to a decentralised model that is empowering more business users also drives the need for a governed data discovery approach (Sallam et al., 2015).

The number of vendors in this market segment increases and points to the increasing demand for BI software that is user-friendly and cost efficient. A more decentralised approach to the use of BI tools demands data preparation tools that are available for business users and analysts. BI and analytics vendors offer self-service data preparation tools to expand the use of analytics (Sallam et al., 2015).

Gartner defined self-service business intelligence as "end users designing and deploying their own reports and analyses within an approved and supported architecture and tools portfolio" (Gartner, 2015). This definition implies controlled self-service business intelligence that maintains co-operation between end users and information technology (IT) specialists. This cooperation ensures quality control, data integrity, and security. Self-service BI business users sometimes are called "power users".
Self-service is relevant because a centralised BI environment is not always able to quickly meet the needs of business users. If a new target is related to a one-time analysis, and the result is needed urgently, it is much more effective if the analysis is performed independently without intermediaries.

Gartner analysts indicate a trend of increasing demand for business-oriented platforms, despite their narrow capabilities unlike IT-centric (BI management-centric) platforms. Thus, the absolute leader in business intelligence and analytics platforms by Gartner Magic Quadrant is Tableau Inc. The popularity of Tableau is due to ease of deployment, simplicity of interface, and insignificant costs. Among the leaders are also Qlik and Microsoft (Sallam et al., 2015).

Qlik provides two products: QlikView and Qlik Sense based on associative architecture in-memory. QlikView is an application development platform and it is intended for IT users to create intuitive and interactive applications with the dashboard. The second product Qlik Sense is a web-based platform that focuses on business users' self-service (Qlik, 2015).

Microsoft provides a wide range of BI and analytics, both centralised and decentralised. One of such business-oriented solutions is Power BI. Another one is SQL Server Analysis Services that support OLAP cubes and data mining technologies. Self-service capabilities are provided by Power BI for Office 365 that is implemented as a cloud service, and also by Power Query, Power Pivot, Power View tools that are supported by Microsoft Excel 2013.

\section{CONCLUSION}

The large volume and variety of data have an impact on the development of business data analysis technologies. The number of business information users has increased according to new tasks that can be solved by means of BI technology solutions.

Big data demands more extensive improvement in technologies for collecting, storing, processing and analysing data. Vendors offer centralised advanced analytics platforms that can be deployed on servers within organisations or in a cloud. Such systems usually require the involvement of IT specialists to implement the system and to prepare analytical information to the end user.

At the same time, there is a growing demand from business users for accessing directly data and data analysis capabilities without intermediaries (IT specialists). One of the requirements for such products is the ease of system deployment and the ease of usage.

The first generation of BI applications ensured the selection of data from relational databases and data transformation, creating the multidimensional databases (OLAP cubes and QlikView analytic cloud), data presentation and visualization tools, as well as offered integration tools with corporate information systems. The next BI application development stage makes it possible to use Data Mining technologies and Predictive Modelling methods.

The second generation of BI systems are focused on the Internet and cloud computing technologies. Innovation continues to develop in areas that provide more intuitive and rapid BI system implementation for the use in data sources of large amounts. 
There is expansion of new products for the business data analysis with the focus on an interactive data analysis. Vendors offer centralised as well as decentralised BI and analytics platforms. They respond to the trend of demand and offer tools for self-service data discovery. New vendors more specialise in the development of self-service products for the data analysis.

\section{REFERENCES}

Brown, M. (2012), Data mining techniques Retrieved Oct. 1, 2015, from http:// www.ibm.com/developerworks/library/ba-data-mining-techniques/ba-data-mining-techniques-pdf.pdf

Codd, E., F. (1979) Extending the database relational model to capture more meaning, Journal ACM Transactions on Database Systems (TODS) TODS Homepage archive, 4 (4), Dec. 1979, 397-434 Retrieved from Oct. 1, 2015, from http://dl.acm org/citation.cfm?id=320109\&dl=ACM\&coll=DL\&C$\mathrm{FID}=552595654 \& \mathrm{CFTOKEN}=50237482$

Gartner Group (2015). IT Glossary, Retrieved Oct. 1, 2015 from http://www. gartner.com/it-glossary/business-intelligence-bi/

Herschel, G., Linden, A., Kart L. (2015) Magic Ouadrant for Advanced Analytics Platforms, Gartner, Retrieved Oct. 1, 2015, from http://www.gartner. $\mathrm{com} /$ technology/reprints.do?id=1-2AHPOU0\&ct $=150225 \& \mathrm{st}=\mathrm{sb}$

IBM (2015). DB2 for Linux, UNIX and Windows. Retrieved Oct. 1, 2015, from: http://www-01.ibm.com/software/data/db2/linux-unix-windows/warehouse-analytics

IBM Corporation (2011) IBM SPSS Modeler 14.2 Modeling Nodes Retrieved Oct. 1, 2015, from ftp://ftp.software.ibm.com/software/analytics/spss/documentation/modeler/14.2/en/ModelingNodes.pdf

IBM Corporation (2013). The strategic advantage of OLAP and multidimensional analysis. Retrieved Oct. 1, 2015, from http://www-01.ibm.com/com$\mathrm{mon} / \mathrm{ssi} / \mathrm{cgi}$-bin/ssialias? infotype=SA\&subtype $=$ WH\&htmlfid=YTW03161CAEN

McKendrick, J. (2012) Opening Up Business Intelligence to the Enterprise: 2012 Survey of Data Professionals on Self-Service BI and Analytics, Retrieved June. 04, 2015, from http://www.unisphereresearch.com/Issues/5374-Opening-Up-Business-Intelligence-to-the-Enterprise-2012-Survey-of-Data-Professionals-on-Self-Service-BI-and-Analytics.htm

Microsoft (2011) Data warehousing, OLAP, and Analysis Services for Share Point 2010 Retrieved Aug. 2011, from https://technet.microsoft.com/en-us/ library/ff696762\%28v=office. $14 \% 29$.aspx

Microsoft (2015 a) Books Online for SQL Server 2016, SQL Server Integration Services Retrieved Oct. 1, 2015, from https://msdn.microsoft.com/en-us/ library/ms141026.aspx

Microsoft (2015 b) Books Online for SQL Server 2016, Data Mining Algorithms Retrieved Oct. 1, 2015, from https://msdn.microsoft.com/en-us/library/ms175595.aspx

Microsoft System Center (2015). Understanding OLAP Cubes Retrieved Oct. 1, 2015, from https://technet.microsoft.com/en-US/library/hh916543.aspx

Oracle (2014). Agile Advanced Analytic Platform (A3P), An Oracle White Paper, October 2014, Retrieved Oct. 1, 2015, from http://www.oracle.com/ technetwork/database/options/advanced-analytics/a3poct2014-2354088.pdf

Oracle (2015). Oracle Database Online Documentation 12c Release 1 (12.1) Data Warehousing and Business Intelligence, Retrieved Oct. 1, 2015, from http://docs.oracle.com/database/121/nav/portal_6.htm

Qlik (2014) What makes QlikView unique, A Qlik $\bar{W}$ hite Paper Retrieved Oct. 1, 2015, from http://www.qlik.com/us/explore/resources/whitepapers/whatmakes-qlikview-unique

Qlik (2015) For developers, Retrieved Oct. 1, 2015, from http://www.qlik.com/ for-developers

Sallam, R. L., Hostmann, B., Schlegel, K., Tapadinhas, J., Parenteau, J., Oestreich, T. W. (2015) Magic Quadrant for Business Intelligence and Analytics Platforms, Retrieved Oct. 1, 2015, from http://www.gartner.com/technology/ reprints.do?id=1-2ACLP1P\&ct $=150220 \& \mathrm{st}=\mathrm{sb}$

Sallam, R. L., Parenteau, J., Hostmann, B., Schlegel, K., Oestreich T. W., Tapadinhas, J., Howson, C. (2015) Critical Capabilities for Business Intelligence and Analytics Platforms, Retrieved Oct. 1, 2015, from https://www. gartner.com/doc/3050717/critical-capabilities-business-intelligence-analytics

SAS (2015) Advanced Analytics Retrieved Oct. 1, 2015, from http://www.sas com/en_us/software/analytics.html\#view-all-products

Taylor, K. L. (2014). Oracle Data Mining Concepts Retrieved Oct. 1, 2015, from https://docs.oracle.com/database/121/DMCON/E17692-15.pdf

Unisphere Research (2015) The future of data warehousing: 2015 Retrieved April 01, 2015, from http://www.unisphereresearch.com/Issues/5238-TheFuture-of-Data-Warehousing-2015.htm

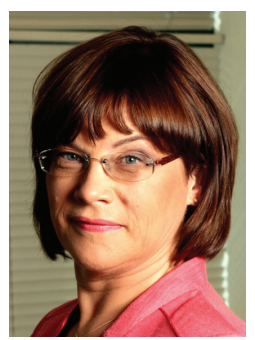

Signe Bāliṇa obtained Doctor degree in Economics in 2002. At present, she is a Professor at the University of Latvia, Faculty of Economics and Management. Since 2009, Signe Bāliņa has been a President of the Latvian Information and Communications Technology Association (LIKTA) that is a professional association uniting organisations of ICT industry, research and educational institutions, as well as ICT professionals. Main objectives of LIKTA are development of information society and growth of ICT industry. Since 2010, Signe Bāliṇa has been a Chairperson of the Board of "IT Competence Centre" that promotes a long-term cooperation between ICT enterprises and science institutions in the fields of natural language technologies and business process analysis technologies.

E-mail: signe.balina@lu.lv

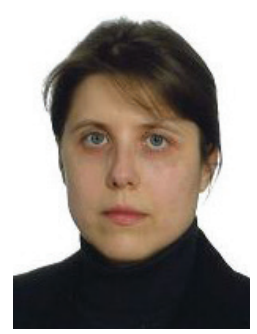

Rita Žuka obtained Master's degree in Social Sciences in 2003. At present, she is a Lecturer at the University of Latvia, Faculty of Economics and Management. Research interests include database design, data analytics, data mining, predictive analytics.

E-mail: rita.zuka@lu.lv

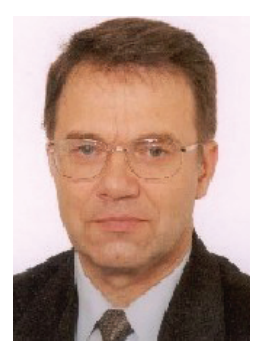

Juris Krasts obtained Master's degree in Computer Sciences in 1995. At present, he is a Lecturer at the University of Latvia, Faculty of Economics and Management. Research interests include business intelligence technology, analysis of business data, predictive analytics and data mining.

E-mail: juris.krasts@1u.lv 